The INL is a

U.S. Department of Energy

National Laboratory

operated by

Battelle Energy Alliance

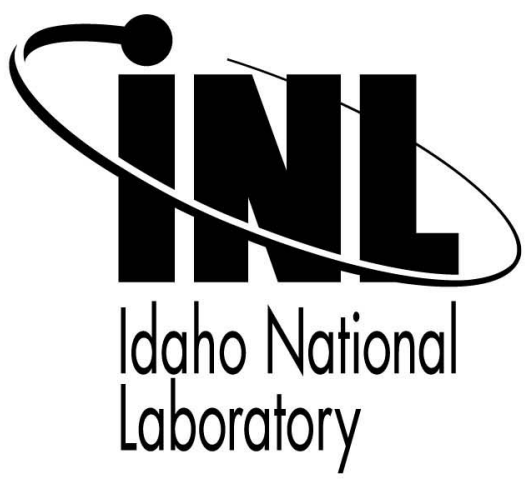

INL/CON-08-14411

PREPRINT

\section{Fabrication Process and Product Quality Improvements in Advanced Gas Reactor UCO Kernels}

\section{ASME $4^{\text {th }}$ International Topical Meeting on High Temperature Reactor Technology}

Charles M. Barnes

W. C. (Clay) Richardson

DeWayne Husser

Matthias Ebner

\section{September 2008}

This is a preprint of a paper intended for publication in a journal or proceedings. Since changes may be made before publication, this preprint should not be cited or reproduced without permission of the author. This document was prepared as an account of work sponsored by an agency of the United States Government. Neither the United States Government nor any agency thereof, or any of their employees, makes any warranty, expressed or implied, or assumes any legal liability or responsibility for any third party's use, or the results of such use, of any information, apparatus, product or process disclosed in this report, or represents that its use by such third party would not infringe privately owned rights. The views expressed in this paper are not necessarily those of the United States Government or the sponsoring agency. 


\section{FABRICATION PROCESS AND PRODUCT QUALITY IMPROVEMENTS IN ADVANCED GAS REACTOR UCO KERNELS}

\author{
Charles M. Barnes \\ Idaho National Laboratory \\ Idaho Falls, Idaho, USA \\ DeWayne Husser \\ Babcock \& Wilcox \\ Lynchburg, Virginia, USA
}

\author{
W. C. (Clay) Richardson \\ Babcock \& Wilcox \\ Lynchburg, Virginia, USA \\ Matthias Ebner \\ Idaho National Laboratory \\ Idaho Falls, Idaho, USA
}

\section{ABSTRACT}

A major element of the Next Generation Nuclear Plant (NGNP)/Advanced Gas Reactor (AGR) Fuel Development and Qualification Program is developing fuel fabrication processes to produce high quality uranium-containing fuel kernels, TRISO-coated particles and fuel compacts needed for planned irradiation tests. The goals of the program also include developing the fabrication technology to mass produce this fuel at low cost. Kernels for the first AGR test, AGR-1, consisted of uranium oxycarbide (UCO) microspheres that were produced by an internal gelation process followed by high temperature steps to convert the $\mathrm{UO}_{3}+\mathrm{C}$ "green" microspheres to $\mathrm{UO}_{2}+\mathrm{UC}_{\mathrm{x}}$. The high temperature steps also densified the kernels.

Babcock and Wilcox (B\&W) fabricated UCO kernels in their Lynchburg facility for the AGR-1 irradiation experiment, which went into the Advanced Test Reactor (ATR) at Idaho National Laboratory in December 2006. An evaluation of the kernel process prior and after these kernels were produced led to several recommendations to improve the fabrication process. These recommendations included testing alternative methods of dispersing carbon during broth preparation, evaluating the method of broth mixing, optimizing the broth chemistry, optimizing sintering conditions, and demonstrating fabrication of larger diameter UCO kernels needed for the second AGR irradiation test, AGR-2.

Based on these recommendations and requirements, a test program was defined and performed. Certain portions of the test program were performed by Oak Ridge National Laboratory (ORNL), while tests at larger scale were performed by $\mathrm{B} \& \mathrm{~W}$. The tests at $\mathrm{B} \& \mathrm{~W}$ have demonstrated improvements in both kernel properties and process operation. Changes in the form of carbon black used and the method of mixing the carbon prior to forming kernels led to improvements in the phase distribution in the sintered kernels, greater consistency in kernel properties, a reduction in forming run time, and simplifications to the forming process. Process parameter variation tests in both forming and sintering steps led to an increased understanding of the acceptable ranges for process parameters and additional reduction in required operating times. Another result of this test program was to double the kernel production rate. Following the development tests, approximately $40 \mathrm{~kg}$ of natural uranium UCO kernels have been produced for use in coater scale up tests, and approximately $10 \mathrm{~kg}$ of low enriched uranium UCO kernels for use in the AGR-2 experiment.

\section{INTRODUCTION}

The goals of the AGR/NGNP Fuel Development and Qualification Program include providing a baseline fuel qualification data set in support of licensing and operation of a Next Generation Nuclear Plant and in support of the near term deployment of a nuclear plant for hydrogen and energy production in the United States. The fuel qualification data will help to reduce the market entry risks posed by technical uncertainties associated with fuel production and qualification [1]. A major element of the program is developing the fuel fabrication processes to produce high quality kernels, TRISOcoated particles and the fuel compacts needed for planned irradiation tests, as well as developing the fabrication technology to mass produce this fuel at low cost. 
At this time coated particle fuels containing either UCO or $\mathrm{UO}_{2}$ are being considered for the NGNP. The AGR-1 fuel kernel was mixed uranium oxide-uranium carbide $\left(\mathrm{UO}_{2}-\mathrm{UC}_{\mathrm{x}}\right.$, often written as "UCO" for convenience). Specifications for AGR fuel kernels include the kernel diameter, aspect ratio (ratio of maximum to minimum diameter), density, uranium enrichment, total uranium content, carbon to uranium ratio, oxygen to uranium ratio and maximum levels for 15 impurities.

Kernel fabrication for the AGR/NGNP Fuel Development and Qualification Program began in early FY 2004, with the initial objective of producing natural uranium UCO kernels for use in small-scale coater development tests at Oak Ridge National Laboratory (ORNL). While kernels of that first production campaign met all specifications, the oxygen to uranium ratio of these kernels was only slightly above the specified minimum value. A series of tests to improve the chemistry of the kernels was initiated in mid FY 2004 [2, 3]. Increasing the ratio of carbon black to uranium in the broth improved the final sintered kernel chemistry. However, during the course of these tests, the density of the sintered kernels degraded, and led to a short term test program to investigate the effects of sintering conditions and methods of dispersing carbon in the broth on final sintered kernel properties.

Once conditions were found that consistently produced kernels with acceptable chemistry and density, kernels were fabricated for use in AGR-1 fuel. Following successful fabrication of $350-\mu \mathrm{m}$ AGR-1 kernels, a longer term test program explored (a) additional improvements in carbon dispersion, (b) adjusting the broth chemistry to optimize the green kernel strength and morphology and (c) reevaluating sintering parameters using kernels made with the optimum carbon black and dispersion methods. Following process improvements found through these tests, production of larger diameter $(425-\mu \mathrm{m})$ kernels for the AGR-2 test was demonstrated.

\section{AGR-1 KERNEL FABRICATION PROCESS}

A diagram of the process used to produce kernels for AGR-1 fuel is shown in Figure 1.

The first step in the kernel fabrication process is preparation of acid-deficient uranyl nitrate (ADUN) by dissolving a high purity uranium feed material in nitric acid. To achieve the specified ${ }^{235} \mathrm{U}$ enrichment, $\mathrm{B} \& \mathrm{~W}$ prepared and blended batches of natural and enriched uranium ADUN. The ADUN was then stored until needed for the further processing in "forming runs."

Kernel forming includes several steps. For the process used for AGR-1 kernels, carbon black, Tamol ${ }^{\mathrm{TM}}$ and a urea solution were mixed with the ADUN solution and then hexamethylene tetraamine (HMTA) added to this mixture to produce the "broth." This broth was then forced through vibrating orifices to form spherical droplets of very uniform diameter. The droplets settled in a column of trichloroethylene (TCE), which serves as a heat transfer fluid. The interfacial tension between the TCE and the aqueous droplets spheridized the droplets, while heat from TCE initiated thermal decomposition of urea to ammonia and $\mathrm{CO}_{2}$. The heating of the broth droplets decomposed the HMTA to $\mathrm{NH}_{3}$, which initiates the gelation process. Decomposition of urea, which is used to complex and stabilize the ADUN to prevent premature precipitation, produced more ammonia. Its role is to complex and stabilize $\mathrm{UO}_{2}{ }^{+2}$ and accelerate the decomposition of HMTA. The gelled kernels were then washed, dried, screened and sampled.

The "green" kernels were heated in a fluidized bed for calcination, carbothermic reduction and sintering. Calcination was carried out in $100 \%$ hydrogen at a moderate temperature and removed the water of hydration from the oxide matrix of the green kernel, decomposed the uranium oxyhydroxide to $\mathrm{UO}_{3}$, and reduced $\mathrm{UO}_{3}$ to $\mathrm{UO}_{2}$. For the carbothermic reduction step, the temperature was increased to $1680^{\circ} \mathrm{C}$ and atmosphere changed to a mixture of argon and $\mathrm{CO}$. Carbon present in the kernel reacts with $\mathrm{UO}_{2}$ to form $\mathrm{UC}_{2}, \mathrm{UC}$ and CO. In the third step of the conversion process, the temperature was increased again to densify the kernels. Following cooling the kernels were removed from the furnace, sieved to remove undersize and oversize kernels, sorted on an inclined table to remove non-spherical kernels and then sampled and analyzed to ensure compliance with specifications.

Seven batches of kernels were composited into a $4.42 \mathrm{~kg}$ lot for use in the AGR-1 tests. Table 1 shows a summary of properties for these kernels, and Figures 2 and 3 show photographs of sectioned and loose AGR-1 kernels. 


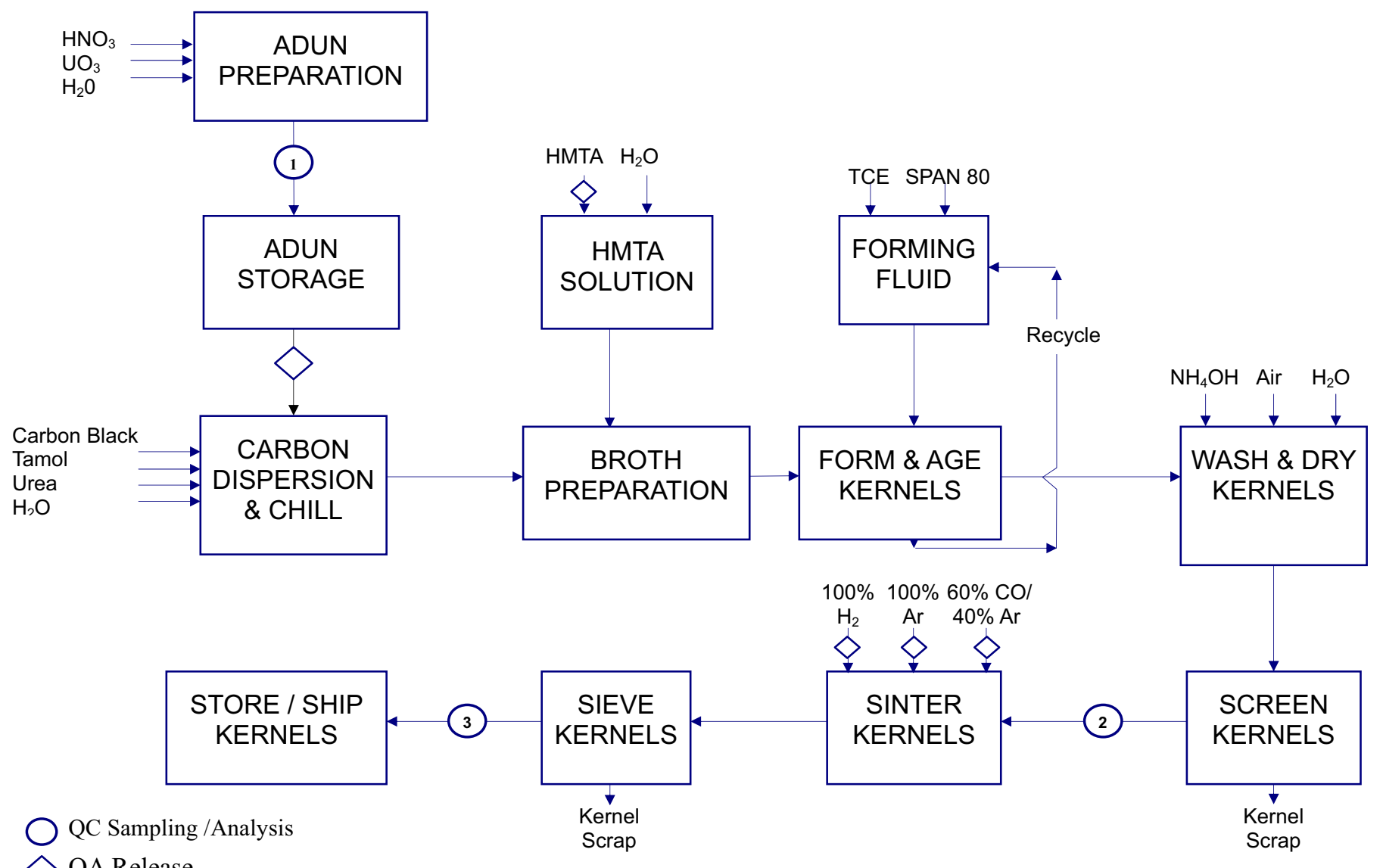

Figure 1. AGR-1 Kernel Fabrication Process [2].

Table 1. AGR-1 Kernel Composite Properties [4].

\begin{tabular}{|l|c|c|}
\hline & Specification & Actual \\
\hline Diameter, $\mu \mathrm{m}$, average & $350 \pm 10$ & 349.5 \\
\hline Diameter, 95\% upper confidence limit & $\leq 360$ & 351.9 \\
\hline Diameter, 95\% lower confidence limit & $\geq 340$ & 347.1 \\
\hline Diameter, 95\%/99\% upper tolerance limit & $\leq 400$ & 375.7 \\
\hline Diameter, 95\%/99\% lower tolerance limit & $\geq 300$ & 323.3 \\
\hline Density, g/cm3 & $\geq 10.5$ & 10.66 \\
\hline U-235 enrichment & $19.8 \pm 0.1$ & 19.74 \\
\hline Total uranium & $\geq 87.0 \%$ & $90.06 \%$ \\
\hline C/U ratio & $0.5 \pm 0.2$ & 0.325 \\
\hline C/U, 95\% upper confidence limit & $\leq 0.7$ & 0.328 \\
\hline C/U, 95\% lower confidence limit & $\geq 0.3$ & 0.323 \\
\hline O/U ratio & $1.5 \pm 0.2$ & 1.36 \\
\hline O/U, 95\% upper confidence limit & $\leq 1.7$ & 1.37 \\
\hline O/U, 95\% lower confidence limit & $\geq 1.3$ & 1.36 \\
\hline Aspect ratio, mean & none & 1.013 \\
\hline Aspect ratio & $\leq 7$ kernels out of 142 with ratio $\geq 1.05$ & 7 kernels out of 142 with ratio $\geq 1.05$ \\
\hline
\end{tabular}




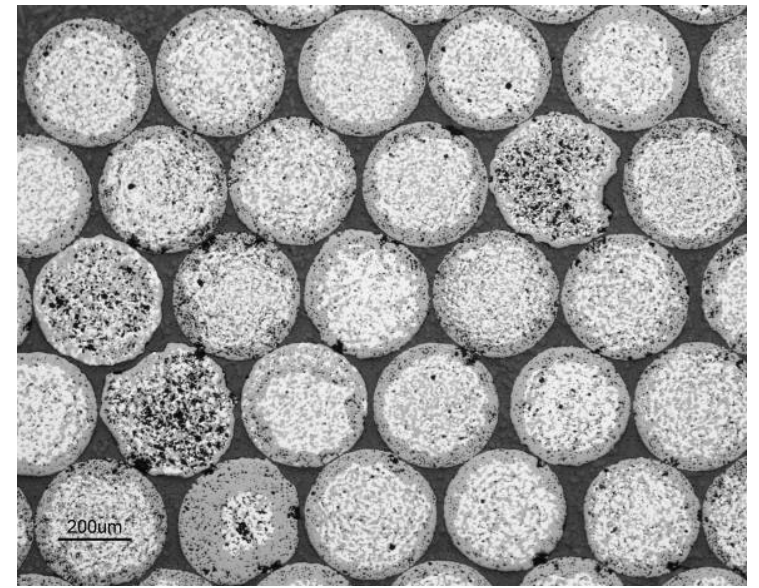

Figure 2. Ceramographic images of AGR-1 kernels.

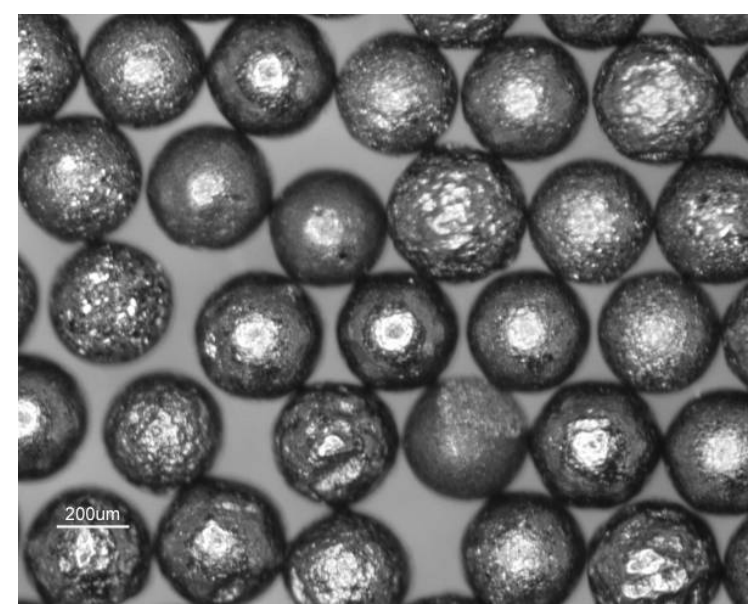

Figure 3. Loose AGR-1 kernels.

While Table 1 shows that the kernels met all specifications, the photos show variability in both kernel surface and internal appearance, suggesting possible kernel to kernel variation in chemistry, density and other properties. The primary goal of subsequent process tests was to improve uniformity both from batch to batch and within a batch.

\section{CARBON DISPERSION}

An earlier kernel fabrication study [3] strongly suggested that control of kernel density and composition would require improving the dispersion of the carbon black during the forming process. Toward this end tests were designed to (a) evaluate dispersion of the carbon black in HMTA rather than ADUN, (b) optimize the surfactant to carbon black ratio, (c) evaluate other dispersants, and (d) evaluate other carbon blacks. Based on characterization and small-scale tests of different carbon blacks and surfactants [5-6], several carbon blacks were selected for testing in the $\mathrm{B} \& \mathrm{~W}$ production line. Raven 1000, a carbon black manufactured by Columbian Chemical Company, had been used in fabrication of kernels for AGR-1 fuel and was included in the tests. The other carbons were prototypes of surface-modified carbons developed by Cabot Corporation. Labeled as type "F" and "G", the Cabot carbons were designed with anionic hydrophilic functionality on their surface, for use without surfactants. Type $\mathrm{F}$ was modified by the grafting of phenylcarboxylic acid groups onto the carbon, while type G has phenylsulfonate groups.

In initial tests at $\mathrm{B} \& \mathrm{~W}$, dispersant behavior of types $\mathrm{F}$ and $\mathrm{G}$ in both acidic and basic solutions was compared using five different mixing methods. The acidic solution, intended to simulate the ADUN solution, consisted of approximately 3.2 $\mathrm{M}$ urea in water that was acidified with nitric acid to $\mathrm{pH} 2$. The basic solution consisted of approximately 3.2 M HMTA and 3.2 $\mathrm{M}$ urea in water, with no acidification. The dispersion trials used five mixing methods: off-line vortex mixing by magnetic stirrer; in-line impeller agitation; in-line mechanical shear pump mixing; in-line ultrasonic shear; and a combination of in-line mechanical shear and ultrasonic shear.

Cabot Type $\mathrm{G}$ carbon black wet and dispersed readily in the $\mathrm{B} \& \mathrm{~W}$ aqueous tests, while type $\mathrm{F}$ did not disperse well. While impeller and vortex mixing easily wet and dispersed the Type $G$ carbon, the best results were obtained with the mechanical shear pump. The mechanical shear pump readily wet and dispersed the carbon within the first few minutes after immersion, but the quality of the dispersion continued to improve steadily and reached the optimum after 30 to 40 minutes of shear action, as monitored by optical microscopy of the mixture. At that point, optical microscopy indicated the complete dispersion of the carbon, to particle sizes on the order of one micron and less. Images of the dispersion in simulant solution taken at 0 and 40 minutes are shown in Figures 4 and 5. Figure 4 shows that Cabot $\mathrm{G}$ carbon wets quickly even with no mechanical mixing, and "dispersed" almost spontaneously in the aqueous simulant solution to form agglomerates that were no larger than 5 microns. Figure 5 shows that mechanical shear mixing for 40 minutes further dispersed the small agglomerates to sizes that could not be resolved optically, and were probably much less than 1 micron. The submicron particles, probably carbon aggregates, scattered the illuminating light quite effectively, giving the image a uniform gray cast with no resolvable features.

In the mechanical shear tests, type $G$ carbon black was stable and did not break down to form an oily film. Under the same conditions, type $\mathrm{F}$ carbon dispersed poorly in the simulant solution, with a large population of agglomerates as large as 50 microns still present after 90 minutes of mechanical shear. Type $F$ carbon was much less stable towards mechanical shear than type $\mathrm{G}$, and formed oily films in all of the tests. 
Ultrasonic shear treatment was much less effective than mechanical shear in dispersing Cabot Type G carbon black. Prolonged sonication produced an oily slick on the dispersion, indicating that the ultrasonic energy had disrupted the dispersant molecules that were chemically grafted to the carbon surfaces. The destruction of the surface grafts destabilized the dispersion and tended to re-agglomerate the carbon black.

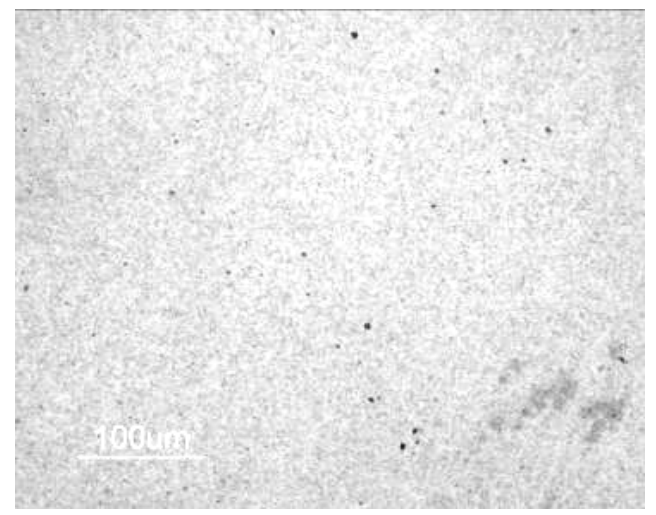

Figure 4. Cabot type $\mathbf{G}$ carbon without mechanical shear mixing in the simulant solution.

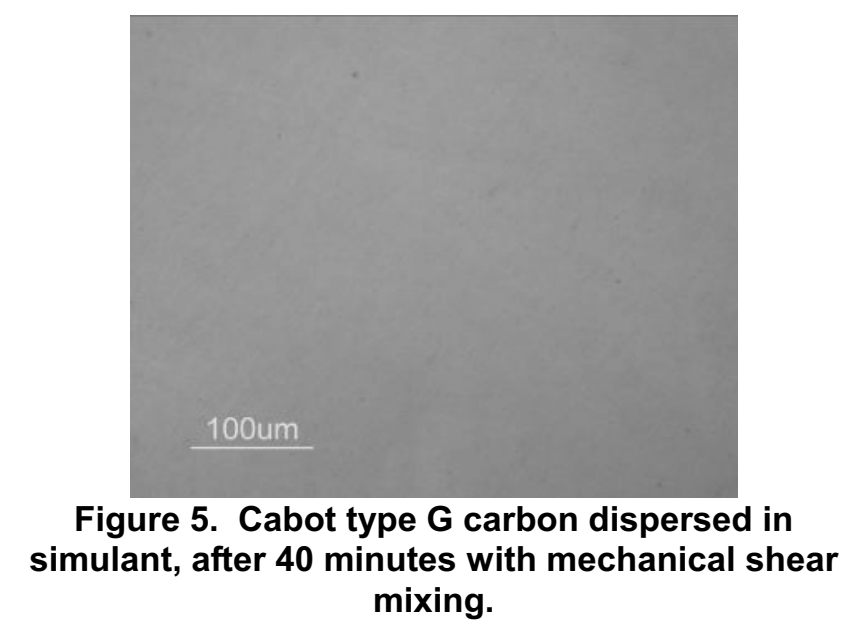

The same five mixing methods were tested in their relative ability to disperse type G carbon in an HMTA-urea solution. Optical microscopy indicated that all five methods produced excellent dispersions within ten minutes of mixing time. An example is shown in Figure 6, which shows the dispersion after vortex mixing (with a magnetic stir bar) in a beaker after 10 minutes. The important feature of Figure 6 is the uniform gray cast of the image, with only a few small resolvable agglomerates visible; the uniform gray cast indicates that almost all of the carbon black was dispersed as submicron particles, probably as individual aggregates. When these dispersions were then acidified to a $\mathrm{pH}$ of 4-6, some reagglomeration was observed, indicating that the dispersion of Cabot G in HMTA-urea solution was not entirely stable upon acidification. Given the results of Type $\mathrm{G}$ carbon in the
HMTA solution, the Type F carbon was tested only using magnetic stirring, and found not to disperse as well as Type G.

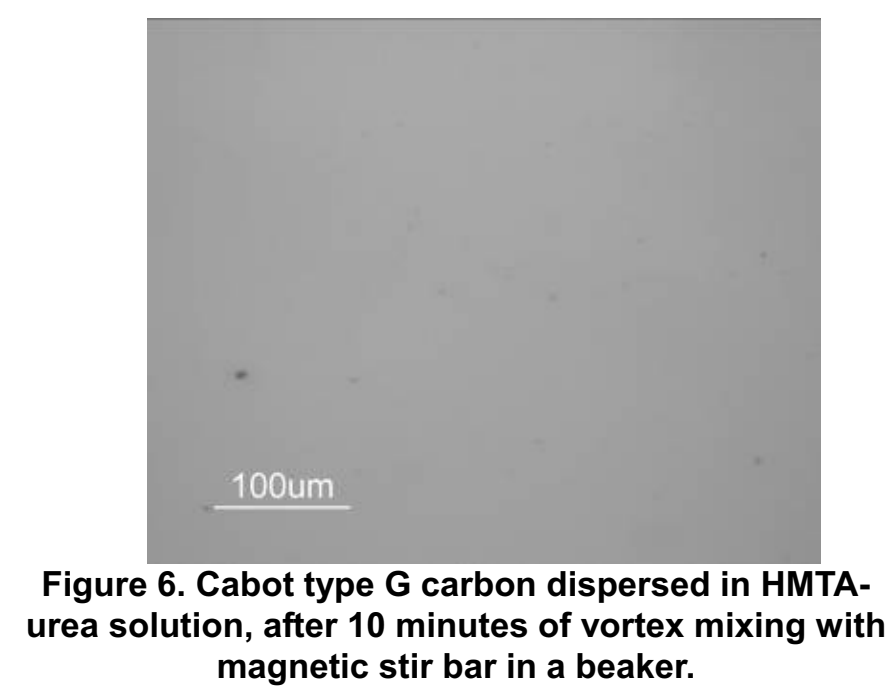

Seven tests were then performed in the B\&W pilot scale kernel fabrication process to compare carbon types and dispersion methods. Forming runs were made using Raven 1000 carbon dispersed in ADUN, Raven 1000 dispersed in HMTA, Cabot Type G carbon dispersed in ADUN, and Cabot Type G dispersed in HMTA. Also, two premixed suspensions of Cabot Type G carbon were tested. Cabot prepared these suspension or slurry forms of Type $\mathrm{G}$ carbon by withdrawing them from their chemical grafting process prior to removal of water and reduction to a powder. The " $\mathrm{B}$ " suspension was purified of reaction byproducts, while the "A" suspension was not purified. Table 2 shows the results for changes on the properties of the sintered kernels. Duplicate runs were made for slurry B. Green kernels from these seven forming tests were sintered at the same conditions, using the sintering conditions used for AGR-1 kernels (see Table 4).

The chemistry for all runs shown in Table 2 was acceptable. The density was good for the case of Cabot Type $\mathrm{G}$ carbon in either solid or slurry form dispersed in HMTA, but marginal or low for the other cases.

Images of sectioned sintered kernels from these tests are shown in Figure 7. Kernels from run 59300 (top left image in Figure 7) show evidence of pullout and some kernels show variations in appearance. Kernels from runs 59315 and 59316 (top right and mid left images) show pores suggesting poor carbon dispersion. Kernels from runs 59318 (bottom left) and 59319 (not shown) have a much more uniform internal structure.

Significant differences can also be seen in surface morphologies of kernels from these runs. As shown in Figure 8 , while the internal appearance of kernels made from Cabot $G$ dispersed in HMTA had a very uniform two-phase 
microstructure, the surface morphology of these kernels varied from smooth, spherical and reflective (59318 in Figure 8) to a non-spherical convoluted appearance (59314 in Figure 8).

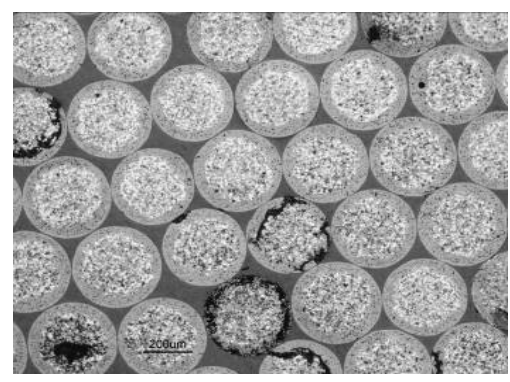

Raven 1000 dispersed in ADUN

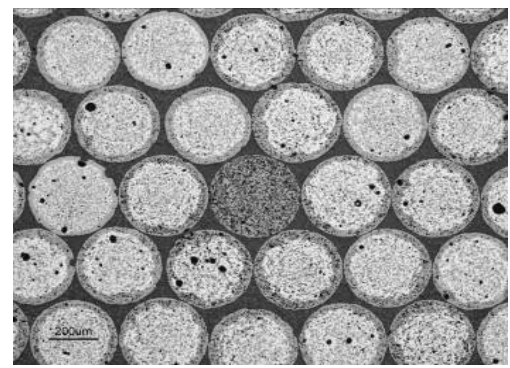

Cabot G dispersed in ADUN

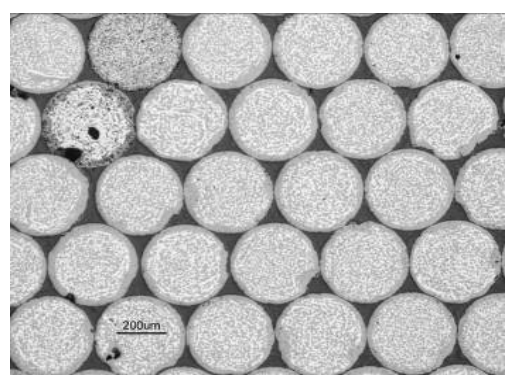

Slurry A dispersed in HMTA
The kernels with the most uniform surface appearance were those from runs 59318 and 59319.

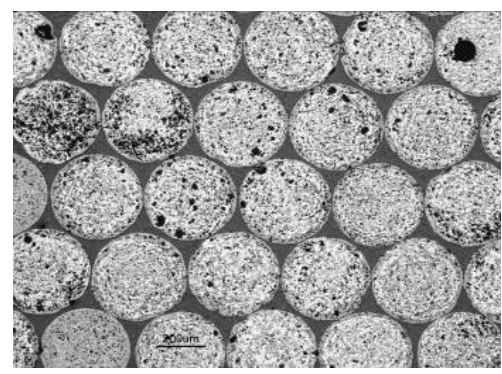

Raven 100 dispersed in HMTA

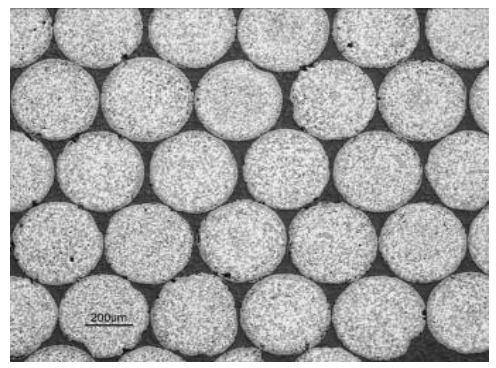

Cabot G dispersed in HMTA

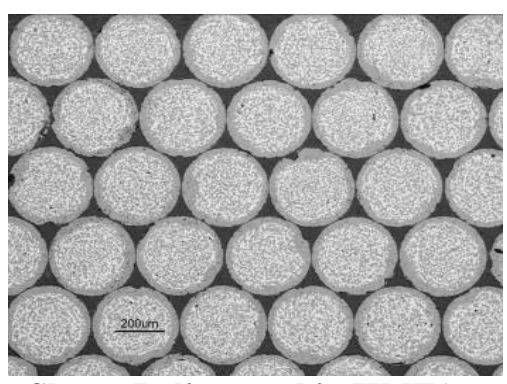

Slurry B dispersed in HMTA

Figure 7. Ceramographic images of kernels from tests 59300, 59315, 59316, 59314, 59317 and 59318.

Table 2. Results of sintering tests at $1890^{\circ} \mathrm{C}$.

\begin{tabular}{|l|c|c|c|c|c|}
\hline \multicolumn{1}{|c|}{ Forming run } & $\begin{array}{c}\text { Sintering run } \\
\text { ID }\end{array}$ & U, wt \% & $\begin{array}{c}\text { O/U Mol } \\
\text { Ratio }\end{array}$ & $\begin{array}{c}\text { C/U Mol } \\
\text { Ratio }\end{array}$ & $\begin{array}{c}\text { Hg } \\
\text { Density, } \\
\text { g/cc }\end{array}$ \\
\hline Raven 1000 in ADUN & 59300 & 89.5 & 1.47 & 0.37 & 10.5 \\
\hline Raven 1000 in HMTA & 59315 & 89.6 & 1.47 & 0.35 & 9.9 \\
\hline Type G carbon in ADUN & 59316 & 89.6 & 1.44 & 0.40 & 10.6 \\
\hline Type G carbon in HMTA & 59314 & 89.6 & 1.47 & 0.36 & 11.0 \\
\hline $\begin{array}{l}\text { Type G carbon suspension in HMTA } \\
\text { ("A" form) }\end{array}$ & 59317 & 89.3 & 1.47 & 0.42 & 11.0 \\
\hline $\begin{array}{l}\text { Type G carbon suspension in HMTA } \\
\text { ("B" form) }\end{array}$ & 59318 & 89.3 & 1.51 & 0.38 & 11.0 \\
\hline $\begin{array}{l}\text { Type G carbon suspension in HMTA } \\
\text { ("B" form) }\end{array}$ & 59319 & 89.4 & 1.49 & 0.36 & 10.9 \\
\hline
\end{tabular}



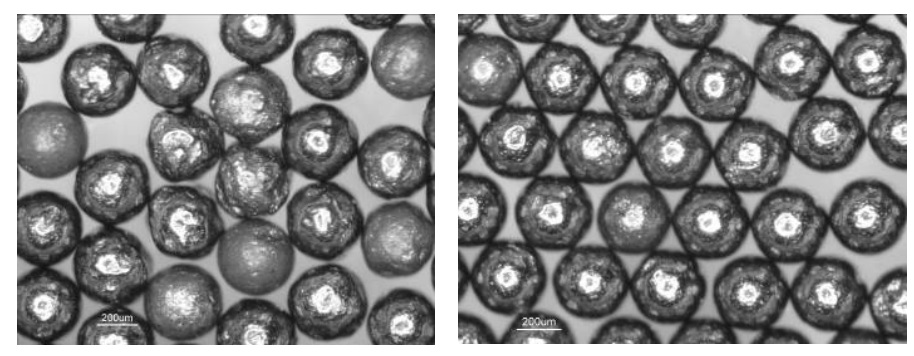

Figure 8. Loose kernels from runs 59314 (left) and 59318 (right).

Based on these results two major changes were made to the $\mathrm{B} \& \mathrm{~W}$ kernel forming process - Raven 1000 carbon black was replaced by Cabot slurry B, and the carbon black was dispersed in HMTA rather than ADUN.

These changes improved the degree of dispersion of carbon in the gelled microspheres, resulting in sintered kernels which had high density and good uniformity. Operationally, the three-hour process of ultrasonic and mechanical shear dispersion of dry carbon powder and dry surfactant in a ventilated enclosure was replaced by mixing the two liquids in a plastic container for about 15 minutes. Less plugging of the droplet forming nozzle, which was probably caused by deposits of agglomerated carbon black, has been encountered using the new surface-modified carbon. The significant reduction in carbon dispersion time translates to cost savings while the improvement in kernel homogeneity translates to improved yields and less waste.

\section{BROTH PARAMETERS}

The primary objective of broth parameter tests was to improve the crush strength and reduce friability of the sintered kernels. A secondary objective was to determine the region of broth parameters for carbon-containing broth that produced green kernels with good hardness and resistance to abrasion or cracking during subsequent processing.

Collins and Hunt have identified key parameters in broth formation for producing strong $\mathrm{UO}_{2}$ kernels [7-8]. For a fixed gel formation temperature and nitrate to uranium ratio, the two key broth parameters are the uranium molarity and the HMTA to uranium ratio. Collins and Hunt found that higher uranium concentrations and HMTA/U ratios produced gelled spheres that were hard and prone to cracking upon drying, while lower uranium concentrations and HMTA/U ratios resulted in gel spheres that were soft and their surfaces prone to abrasion during handling and washing. The proposed "ideal broth zone" for $\mathrm{UO}_{2}$ kernel formation is the region between the two solid lines shown on Figure 9.

Early development and AGR-1 production forming runs of UCO kernels at $\mathrm{B} \& \mathrm{~W}$ had used broth parameters that fell below this ideal broth zone for $\mathrm{UO}_{2}$ kernels. These UCO forming runs were in the region of 1.09-1.22 uranium molarity and HMTA/U ratios of 1.22-1.41. The resultant kernels had poor crush strength and some observable friability. Some later runs used higher uranium molarities and HMTA/U ratios of 1.18-1.32 to bring conditions into the ideal broth zone, producing more robust kernels.

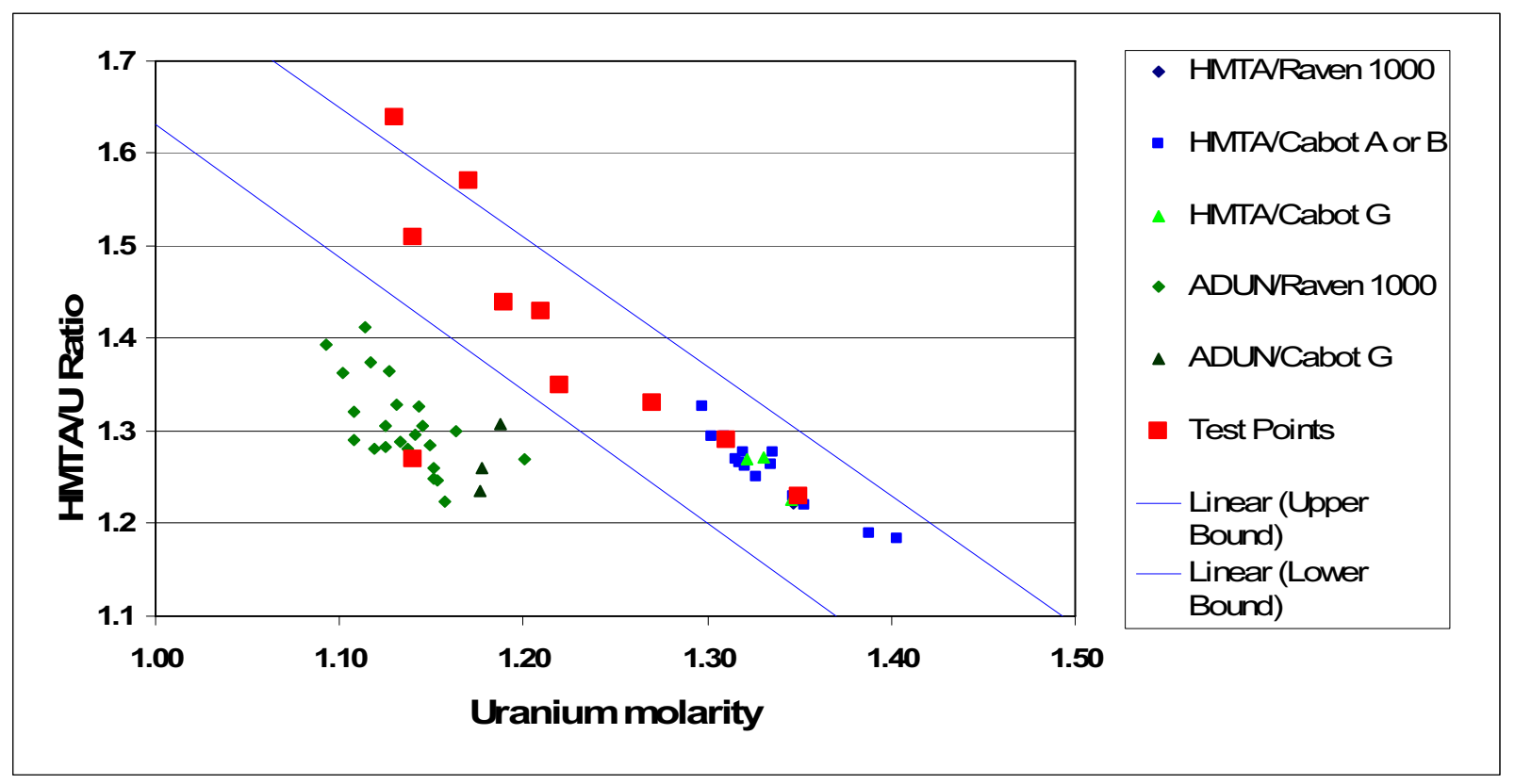

Figure 9. Ideal Broth Zone. 
Broth conditions for ten tests, runs 59308, 59309 and 59330 through 59337, were selected to explore other regions of this ideal broth zone and are shown as red square data points on Figure 9. One point clearly below the lower line and two points above the upper line were selected to better evaluate the boundaries of the ideal zone. All gelled kernel batches were sintered at identical conditions. The measured response parameters included gelled sphere density, sintered kernel density, sintered kernel crush strength, sintered kernel aspect ratio, kernel surface appearance, kernel internal defects (as determined by visual examination of ceramographic mounts of sectioned kernels), and sintered kernel chemistry $(\mathrm{O} / \mathrm{U}$ and $\mathrm{C} / \mathrm{U}$ ratios $)$. For all broth parameter evaluation runs, the Cabot slurry $\mathrm{B}$ was used, with dispersion of carbon in HMTA prior to mixing with ADUN.

The two broth parameters appear to have at most a small effect on kernel crush strength. Figures 10 and 11 show apparent correlations between kernel crush strength and each of the two broth variables. However, the standard deviation of crush strength measurements ranged from $0.43 \mathrm{lbs}$ to $0.84 \mathrm{lbs}$. Error bars shown on Figures 10 and 11 represent one standard deviation. A single factor analysis of variance at a significance level of 0.05 indicates that there is no difference in the crush values for any of the nine batches of particles.

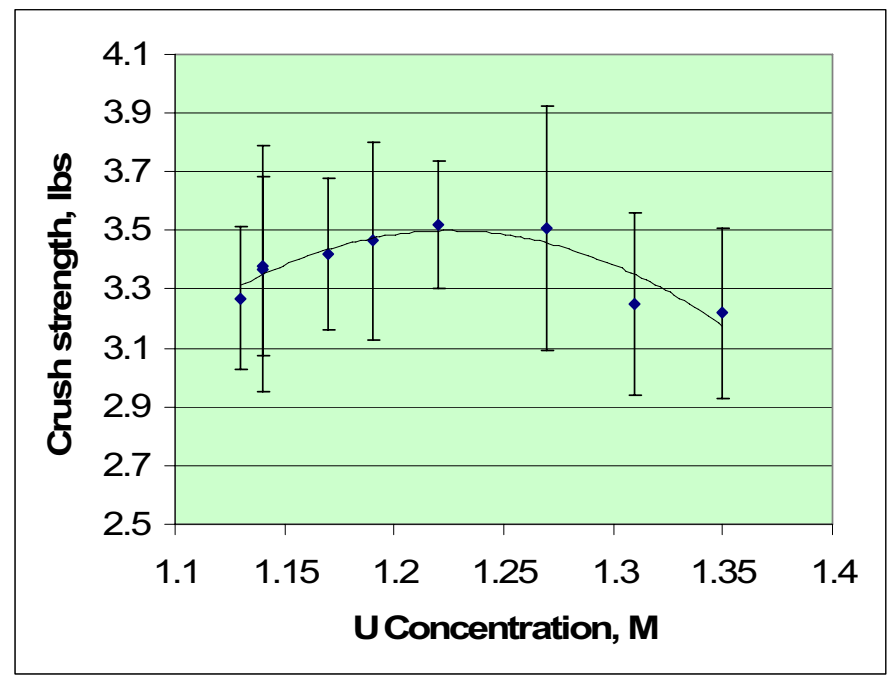

Figure 10. Relationship between $U$ concentration in the broth and kernel crush strength.

Green kernel density decreased at higher broth uranium concentrations and lower HMTA to U ratios. However, there was no correlation between broth parameters and sintered kernel density.

Images of 105-132 loose and sectioned kernels were reviewed to determine surface appearance and internal defects. Identifying surface types and internal defects is somewhat subjective, as appearances can vary within a "type" and defects can vary in size. Also the limited number of kernels examined $(\sim 120)$, makes quantification of defects tentative at best. To minimize this subjectivity, all values reported in Table 3 were determined by the same B\&W engineer. Surface appearance was categorized as type 1 (smooth and highly reflective), type 2 (evenly textured and poorly reflective) and type 3 (bumpy or irregular surface). No batch had more than $1 \%$ (one kernel out of the sample) type 3 kernels. Two batches, 59331 and 59333, were 99-100\% type 2 , while all other batches were predominately type 1 . The broths responsible for the two batches that produced all type 2 kernels had been prepared using conditions outside of the "ideal broth zone" of Figure 9. However, batch 59334, which was also prepared with conditions outside (above) the ideal zone, showed a type 1/type 2 distribution similar to many of the batches prepared with conditions clearly falling inside the ideal zone.

Internal defects were grouped into four categories. Kernels with lens-shaped voids were called "lenticular" defects, while round or nearly round voids were called "void defects". "Pullout" refers to kernels showing dark areas due to erosion or massive grain loss from an area or areas of the surface of a kernel during grinding or polishing. Pullout could also be an indication of friability. The fourth category of internal defects is obvious cracks.

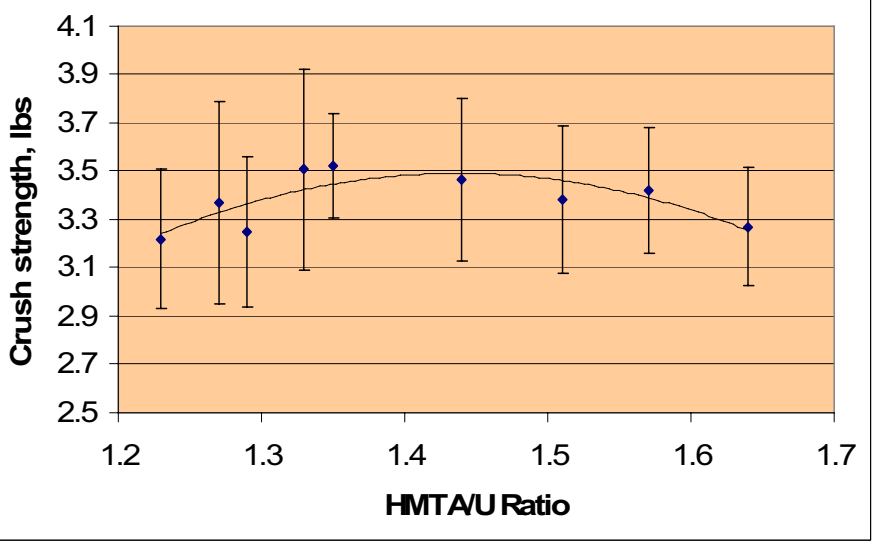

\section{Figure 11. Relationship between broth HMTA/U ratio and kernel crush strength.}

Of the ten test batches shown in Figure 9, the four batches of kernels prepared with the lowest uranium concentrations had no cracks, while for the other batches, up to $2 \%$ of the kernels had cracks. Batch 59334, with the highest HMTA/U ratio, showed the highest amount of pullout, $6 \%$. The data show no clear relationships between broth parameters and lenticular or round voids. Batches 59337 and 59310 had 
relatively low levels of all defects, while only batch 59333 had no kernels with cracks or evidence of pullout.

Sintered kernel mean aspect ratio was found to have a modest relationship to broth $\mathrm{HMTA} / \mathrm{U}$ ratio, while its correlation to broth uranium concentration was poor. The most consistently spherical kernels could be produced at the highest HMTA/U ratios.

As could be expected, sintered kernel $\mathrm{C} / \mathrm{U}$ and $\mathrm{O} / \mathrm{U}$ chemistry showed little to no relationship to broth uranium concentrations or HMTA/U ratios. However, as would also be expected, the broth $\mathrm{C} / \mathrm{U}$ ratio has a very strong effect on kernel chemistry. Figure 12 shows the relationships of kernel chemistry to broth $\mathrm{C} / \mathrm{U}$ ratio.

All kernel batches produced for these runs met kernel specification properties. Also, while there were batch to batch variations in defects and crush strength values, it was found that in general there were no strong correlations between these kernel properties and the broth parameters varied. Thus, it was concluded that a wide range of broth parameters could produce good UCO kernels and that this region was not limited to the "ideal broth zone" found for $\mathrm{UO}_{2}$ kernels.

Table 3. Sintered kernel surface appearance and internal defects.

\begin{tabular}{|c|c|c|c|c|c|c|c|c|c|}
\hline \multirow{2}{*}{ Batch } & \multicolumn{2}{|c|}{ Broth Parameters } & \multicolumn{2}{|c|}{$\begin{array}{c}\text { Sintered Kernel Surface } \\
\text { Appearance }\end{array}$} & \multicolumn{3}{|c|}{ Sintered Kernel Internal Mount Defects } \\
\cline { 2 - 11 } & Uranium, M & $\begin{array}{c}\text { HMT } \\
\text { A/U }\end{array}$ & Type 1 & Type 2 & Type 3 & Lenticular & Void & Cracks & Pullout \\
\hline \hline 59309 & 1.31 & 1.29 & $91 \%$ & $8 \%$ & $1 \%$ & $8 \%$ & $1 \%$ & $2 \%$ & $3 \%$ \\
\hline 59310 & 1.35 & 1.23 & $85 \%$ & $15 \%$ & $0 \%$ & $2 \%$ & $1 \%$ & $2 \%$ & $3 \%$ \\
\hline 59330 & 1.22 & 1.35 & $93 \%$ & $6 \%$ & $1 \%$ & $9 \%$ & $3 \%$ & $2 \%$ & $1 \%$ \\
\hline 59331 & 1.14 & 1.27 & $0 \%$ & $100 \%$ & $0 \%$ & $8 \%$ & $1 \%$ & $0 \%$ & $1 \%$ \\
\hline 59332 & 1.14 & 1.51 & $77 \%$ & $23 \%$ & $0 \%$ & $8 \%$ & $3 \%$ & $0 \%$ & $1 \%$ \\
\hline 59333 & 1.17 & 1.57 & $0 \%$ & $99 \%$ & $1 \%$ & $4 \%$ & $3 \%$ & $0 \%$ & $0 \%$ \\
\hline 59334 & 1.13 & 1.64 & $81 \%$ & $19 \%$ & $0 \%$ & $5 \%$ & $2 \%$ & $0 \%$ & $6 \%$ \\
\hline 59335 & 1.19 & 1.44 & $76 \%$ & $23 \%$ & $0 \%$ & $10 \%$ & $1 \%$ & $1 \%$ & $3 \%$ \\
\hline 59336 & 1.27 & 1.33 & $84 \%$ & $15 \%$ & $1 \%$ & $8 \%$ & $1 \%$ & $1 \%$ & $2 \%$ \\
\hline 59337 & 1.21 & 1.43 & $71 \%$ & $29 \%$ & $0 \%$ & $1 \%$ & $2 \%$ & $1 \%$ & $3 \%$ \\
\hline
\end{tabular}

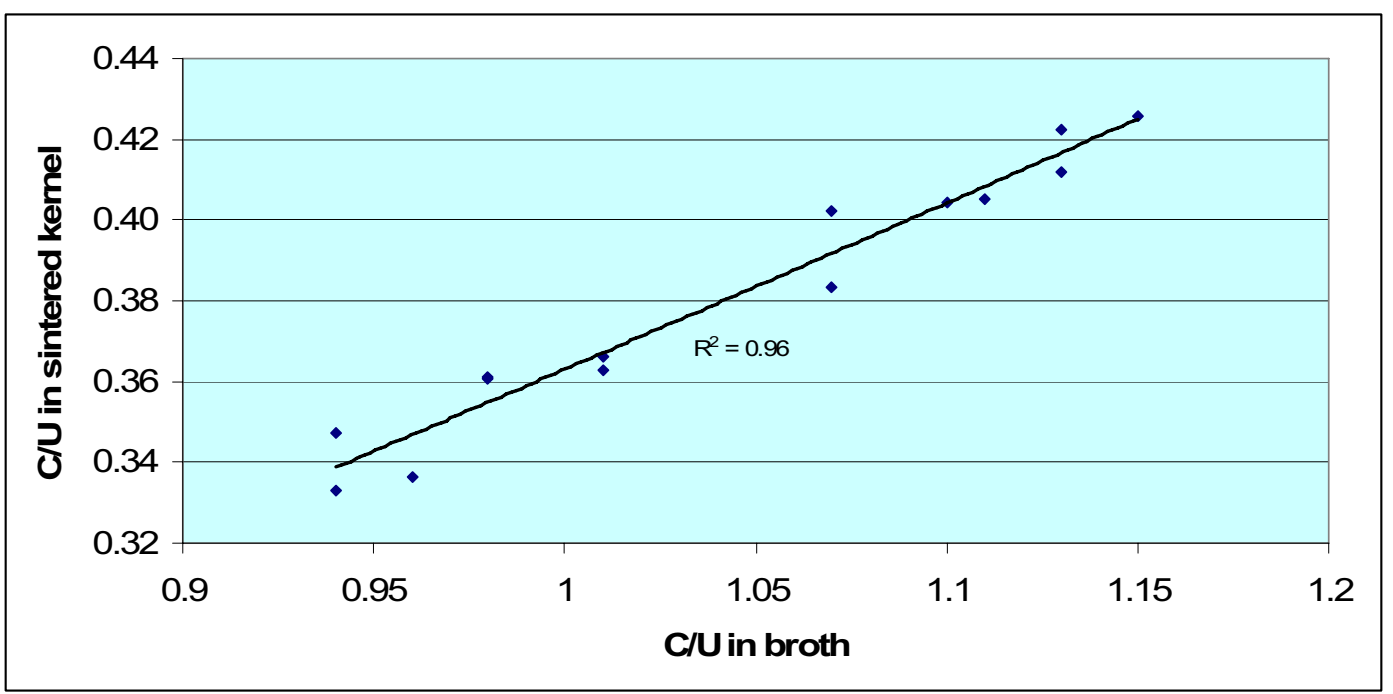

Figure 12. Relationship between sintered kernel $\mathrm{C} / \mathrm{U}$ ratio and broth $\mathrm{C} / \mathrm{U}$ ratio. 


\section{SINTERING PARAMETERS}

The "baseline" sintering schedule, which had been used to produce AGR-1 kernels, is shown in Table 4. Five sintering tests with alternative schedules were performed with green kernels that had been produced using the improved forming process. When the densification temperature was reduced from $1890^{\circ} \mathrm{C}$ to $1800^{\circ} \mathrm{C}$, with the hold time at that temperature being constant at 4 hours, the kernel density dropped from $10.9 \mathrm{~g} / \mathrm{cm}^{3}$ to $9.6 \mathrm{~g} / \mathrm{cm}^{3}$. Reducing the hold time at $1890^{\circ} \mathrm{C}$ to only 10 minutes resulted in a density of $10.0 \mathrm{~g} / \mathrm{cm}^{3}$; however the density of the kernels having a 1-hour hold time was found to be equivalent to those after a 4-hour hold, $10.9 \mathrm{~g} / \mathrm{cm}^{3}$. Reducing the carbothermic hold time from 1 hour in $\mathrm{CO} /$ argon to 35 minutes in argon only had no effect on the sintered kernel density. Based on these results, the one change to the baseline sintering conditions recommended was to reduce the final sintering time from 240 to 60 minutes.

Table 4. Baseline Sintering Conditions.

\begin{tabular}{|l|}
\hline Heatup and $\mathrm{UO}_{3}$ Reduction: \\
$0-100^{\circ} \mathrm{C}$ in $\mathrm{Ar} @ 4^{\circ} \mathrm{C} /$ min \\
$100-550^{\circ} \mathrm{C} \mathrm{H}_{2} @ 4^{\circ} \mathrm{C} / \mathrm{min}$ \\
$550^{\circ} \mathrm{C}-\mathrm{Hold} \sim 4$ min in $\mathrm{H}_{2}$ \\
Switch to $\mathrm{Ar}$. \\
\hline Carbothermic Reduction: \\
$550-1680^{\circ} \mathrm{C}$ in $\mathrm{Ar} @ 40^{\circ} \mathrm{C} / \mathrm{min}$ \\
$1680^{\circ} \mathrm{C}-\mathrm{Hold} 4$ minutes in $\mathrm{Ar}$ \\
$1680^{\circ} \mathrm{C}-$ Switch to $60 / 40 \mathrm{CO} / \mathrm{Ar}$ \\
Hold for 60 minutes \\
\hline Sintering: \\
$1680-1890^{\circ} \mathrm{C}$ in $60 / 40 \mathrm{CO} / \mathrm{Ar} @ 40^{\circ} \mathrm{C} / \mathrm{min}$ \\
$1890^{\circ} \mathrm{C}-\mathrm{Hold}$ for $240 \mathrm{~min}$ in $60 / 40 \mathrm{CO} / \mathrm{Ar}$ \\
$1890^{\circ} \mathrm{C}-$ Switch to $100 \% \mathrm{Ar}$ \\
\hline Cooldown: \\
$1890-\mathrm{RT}$ in $\mathrm{Ar} @$ Power Off \\
\hline
\end{tabular}

\section{IMPROVED PROCESS DEMONSTRATIONS \& AGR-2 KERNEL FABRICATION}

Following the above kernel fabrication process development, approximately $20 \mathrm{~kg}$ of $425-\mu \mathrm{m}$ diameter UCO kernels were produced. Then B\&W's kernel line was idle for approximately 15 months, and restarted in 2008 to produce an additional $20 \mathrm{~kg}$ of natural uranium UCO kernels, followed by approximately $10 \mathrm{~kg}$ of low-enriched uranium UCO kernels that will be used in making fuel for the AGR-2 experiment.

In the first $20-\mathrm{kg}$ campaign, 14 forming and sintering batches were produced. All batches met all kernel specifications. Average batch and composite properties for these kernels are shown in Table 5. Forming process yields for 12 of the 14 runs were $95-99 \%$. Operational issues experienced in the remaining two runs reduced the yield to about $80 \%$.
In each of the first four forming runs, 4 moles of uranium were processed, but the batch size was doubled for the remaining 10 runs. This increase in batch size was possible in large part because of reduced mixing time achieved by use of the Cabot carbon black slurry.

Table 5. UCO Kernel Lot 69304 Properties.

\begin{tabular}{|l|c|c|c|c|}
\hline & \multicolumn{2}{|c|}{ Batches } & \multicolumn{2}{c|}{ Composite } \\
\hline & Average & $\begin{array}{c}\text { Stnd } \\
\text { Dev }\end{array}$ & Average & $\begin{array}{c}\text { Stnd } \\
\text { Dev }\end{array}$ \\
\hline O/U Ratio & 1.48 & 0.026 & 1.48 & 0.005 \\
\hline C/U ratio & 0.367 & 0.028 & 0.365 & 0.005 \\
\hline $\begin{array}{l}\text { Density, } \\
\text { g/cm }\end{array}$ & 10.74 & 0.09 & 10.71 & 0.01 \\
\hline Aspect ratio & 1.005 & 0.0015 & 1.005 & 0.005 \\
\hline $\begin{array}{l}\text { Diameter, } \\
\text { microns }\end{array}$ & 426.7 & 9.2 & 429.0 & 8.9 \\
\hline
\end{tabular}

A gradual decrease in the $\mathrm{C} / \mathrm{U}$ ratio over the first six batches, from 0.37 to 0.34 , prompted an analysis of the carbon slurry feed material. It was found that the fraction of carbon in the slurry was lower than reported by the manufacturer. An adjustment in the feed carbon rate was made for subsequent runs and restored the sintered kernel $\mathrm{C} / \mathrm{U}$ ratio to 0.40 .

During sintering of one of the batches, a coolant leak during the $1680^{\circ} \mathrm{C}$ hold led to a shutdown of the furnace. The furnace was cooled down and the kernels unloaded and held in a nitrogen atmosphere glove box until the leak was fixed. They were then reloaded into the furnace and sintering resumed. Characterization and visual examination of these kernels showed no detrimental effects of the interruption during sintering.

Restart of the kernel line in early 2008 went smoothly for both forming and sintering operations, and no equipment problems were experienced during the campaign to produce another $20 \mathrm{~kg}$ of natural uranium UCO kernels nor $10 \mathrm{~kg}$ of low-enriched uranium UCO kernels. Average properties of these two kernel lots are shown in Table 6. Photographs of loose and mounted kernels from the LEU composite are shown in Figures 13 and 14.

Table 6. UCO Kernels Produced in 2008.

\begin{tabular}{|l|c|c|c|c|}
\hline & \multicolumn{2}{|c|}{$\begin{array}{c}\text { Nat Uranium } \\
\text { UCO Composite }\end{array}$} & \multicolumn{2}{c|}{ LEU UCO Composite } \\
\hline & Average & $\begin{array}{c}\text { Stnd } \\
\text { Dev }\end{array}$ & Average & $\begin{array}{c}\text { Stnd } \\
\text { Dev }\end{array}$ \\
\hline O/U Ratio & 1.47 & 0.01 & 1.43 & 0.005 \\
\hline C/U ratio & 0.39 & 0.005 & 0.39 & 0.002 \\
\hline $\begin{array}{l}\text { Density, } \\
\text { g/cm }\end{array}$ & 10.97 & 0.04 & 10.97 & 0.03 \\
\hline Aspect ratio & 1.010 & 0.004 & 1.012 & 0.006 \\
\hline $\begin{array}{l}\text { Diameter, } \\
\text { microns }\end{array}$ & 421 & 7.1 & 427 & 8.8 \\
\hline
\end{tabular}




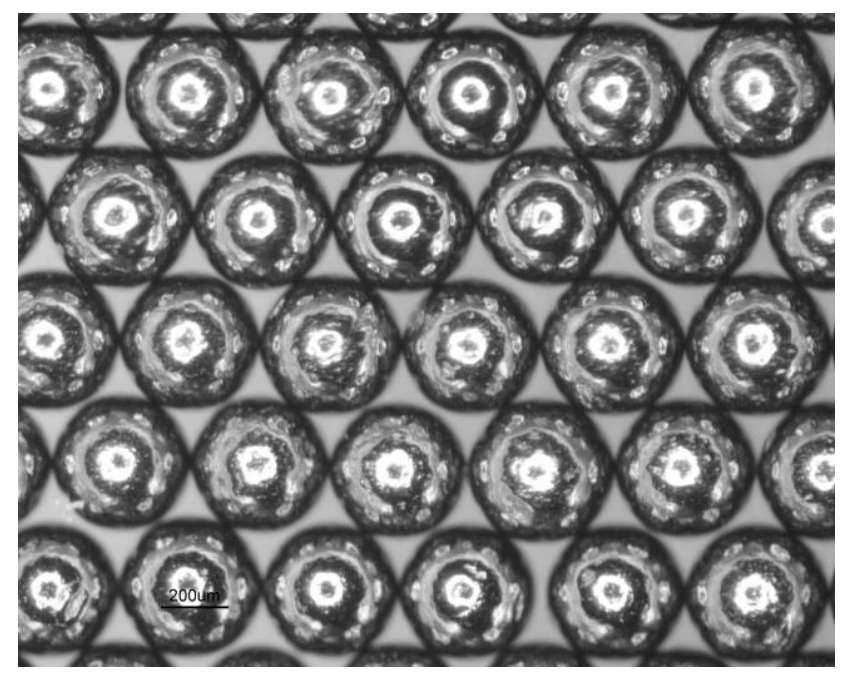

Figure 13. Loose AGR-2 UCO kernels.

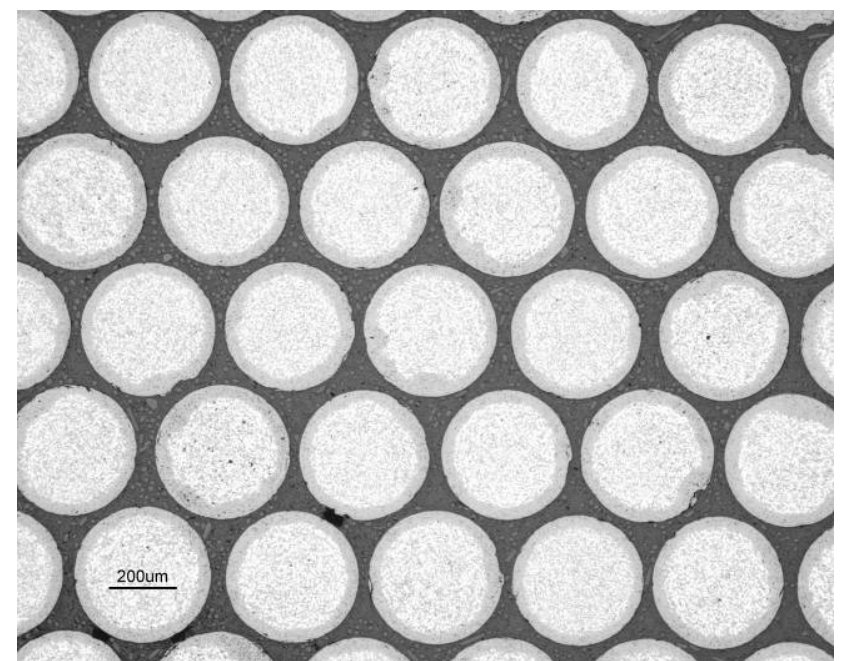

Figure 14. Ceramographic mount of composited AGR-2 kernels.

\section{CONCLUSIONS}

The overall goals of the kernel development program have been achieved. Using a new carbon and a new method of mixing the carbon, carbon dispersions, the resultant kernel quality and process yields were improved. High density kernels with very consistent chemistry have been repeatably produced using the slurry B form of Cabot Type G carbon, and dispersing this carbon in HMTA rather than ADUN. These changes in the forming process also resulted in improvements in process operation, primarily less plugging of forming nozzles and a significant reduction in the forming run time. Reducing the high temperature hold period during sintering from four hours to one hour, perhaps in combination with improved carbon dispersion, also appears to have eliminated kernels with irregular or bumpy surfaces.

Production of larger diameter kernels was accomplished smoothly with minor adjustments to the process.
A systematic variation of key broth parameters has shown that quality kernels can be produced over a wide range of forming conditions. Results from these runs also showed that slight improvements in kernel quality could be achieved by increasing the HMTA to uranium ratio and decreasing the uranium concentration from values that had been used in the past.

Following the development tests, the batch size was doubled during a production campaign with no detrimental effects on kernel properties.

Because of the knowledge gained from the kernel fabrication development tests, there is high confidence that high quality kernels can be produced for all future AGR tests.

\section{ACKNOWLEDGMENTS}

This manuscript has been authored by Battelle Energy Alliance, LLC under Contract No. DE-AC07-05ID14517 with the U.S. Department of Energy. The authors wish to thank the operators at $\mathrm{B} \& \mathrm{~W}$ that were involved in both kernel development and production runs and researchers at ORNL that supported kernel fabrication development. The United States Government retains and the publisher, by accepting the article for publication, acknowledges that the United States Government retains a nonexclusive, paid-up, irrevocable, world-wide license to publish or reproduce the published form of this manuscript, or allow others to do so, for United States Government purposes.

\section{REFERENCES}

[1] Petti, D., Hobbins, R., Kendall, J., Saurwein, J., 2005, Technical Program Plan for the Advanced Gas Reactor Fuel Development and Qualification Program, INL/EXT-05-00465, Revision 1.

[2] Ebner, M. A., 2004, Chemistry Improvement for the Production of LEU UCO Fuel Using Manufacturing Scale Equipment - FY 2004 Final Report, INEEL/EXT-04-02372.

[3] Ebner, M. A., 2005, Chemistry Improvement Tests for the Production of LEU UCO Fuel Using Manufacturing Scale Equipment, FY 2005 Final Report, INL/EXT-05-00618.

[4] BWXT Nuclear Products Division, Data Package for Lot G73D-20-69302, March 11, 2005.

[5] Contescu, C. I., Baker, F. S., Hunt, R. D., Collins, J. L., Burchell, T. D., 2008, "Selection of Water-Dispersible Carbon Black for Fabrication of Uranium Oxicarbide Microspheres," Journal of Nuclear Materials 375, pp. 38-51. 
[6] Contescu, C. I., 2005, Selection and Characterization of Carbon Black and Surfactants for Development of Small Scale Uranium Oxicarbide Kernels, ORNL/TM-2005/548.

[7] Collins, J. L. and Hunt, R. D., 2005, Optimum Process Parameters for Preparations of Ideal $\mathrm{UO}_{2}, U C O$ and (U, $\mathrm{Pu}) \mathrm{O}_{2}$ Kernels by the Internal Gelation Process, ORNL/CF05/07.

[8] Hunt, R. D. Collins, J. L., 2004, "Uranium Kernel Formation via Internal Gelation," Radiochim. Acta 92, pp. 909-915. 